\title{
The Additional Irradiation of the Tumor Bed "The Boost" in the Breast Cancer Conservative Treatment: What Techniques?
}

Ahmedou Toulba*, Iraqi M, Mouhajir N, Nouh M, Diakité A, Nkoua-Epala B, Kebdani T, Elgueddari B and Benjaafar N

Radiotherapy Department, National Institute of Oncology in Rabat, BP 6213 Rabat, Morocco

*Corresponding author: Ahmedou Toulba, Radiotherapy Department, National Institute of Oncology in Rabat, BP 6213 Rabat, Morocco, Tel: 590690837949; E-mail: ahmedoux@gmail.com

Received date: Oct 18, 2013, Accepted date: Jan 08, 2015, Publication date: Jan 15, 2015

Copyright: (C) 2015 Toulba A, et al. This is an open-access article distributed under the terms of the Creative Commons Attribution License, which permits unrestricted use, distribution, and reproduction in any medium, provided the original author and source are credited.

\begin{abstract}
Background: The breast cancer conservative treatment, rise importantly through screening and early diagnosis, neoadjuvant treatment, improved surgical techniques and adjuvant radiotherapy.

The increase dose to the tumor bed after breast irradiation increases the rate of local control. Different techniques are used to deliver the boost to the tumor bed: the direct beam of electrons, photons with reduced tangential fields, low (LDR) and high dose rate (HDR) brachytherapy.
\end{abstract}

Purpose: To compare different boost techniques in local control and cosmetic results in breast cancer.

Materials and methods: A retrospective study through a series of 74 patients treated at the National Institute of Oncology during 2007 for breast cancer and who received additional radiation to the tumor bed after whole breast irradiation.

Results: The median age of patients was 44.5 years (25-59), $81 \%$ were settled and $19 \%$ postmenopausal, $38 \%$ of tumors were stage $\mathrm{T} 1,60 \% \mathrm{~T} 2,1 \% \mathrm{~T} 3,1 \% \mathrm{~T} 4,62 \% \mathrm{~N} 0,37 \% \mathrm{~N} 1$ and $1 \% \mathrm{~N} 2$. For treatment: two patients received neoadjuvant chemotherapy (3\%). Conservative surgery was lumpectomy in 54 cases $(72 \%)$, quadrantectomy in 19(23\%) and zonectomy in one. The oncoplastic remodeling was performed in 24 patients (32\%) and reoperation in 19 patients $(25 \%)$. It was an infiltrative ductal carcinoma in $90 \%$ of cases. Radiotherapy interested the breast and chest wall in 74 cases $(100 \%)$, the supraclavicular fossa in 41 cases $(55 \%)$, the internal mammary chain in $29(39 \%)$ and axilla in $6(8 \%)$. It was 50 Gy in 25 fractions in 53 patients (72\%), 42 Gy in 15 fractions $(2.8 \mathrm{~Gy})$ in 21 patients $(28 \%)$ with median of 37 days (19-60). The additional irradiation of the tumor bed was delivered by electrons at the dose of $15 \mathrm{~Gy}$ in five fractions in $38 \%$ of cases, or by HDR brachytherapy dose of $10 \mathrm{~Gy}$ in two fractions separated by 10 to 12 hours in $34 \%$, or by LDR brachytherapy of $15 \mathrm{~Gy}$ in $4 \%$, or by photons at the dose of $15 \mathrm{~Gy}$ in five fractions (9 patients) or six fractions in $24 \%$ (9 patients). The median time between breast irradiation and the complement was 11 days. The median follow-up was 52 months (3-47 months), 61 patients $(82.5 \%)$ were in situation of good local control, three $(4 \%)$ of local recurrence, 10 cases $(13.5 \%)$ of distant metastases (four were always followed, two died, four lost sight) and nine (12\%) were lost to follow-up. Side effects were type of fibrosis in 28 patients (38\%), telangiectasia in $5(7 \%)$, disorders of skin pigmentation in $11(15 \%)$.

Conclusion: The increase in dose to the tumor bed after conservative treatment of breast cancer allows increasing the rate of local control without compromising too much the aesthetic results. The comparison between the different techniques of boost did not show significant differences. Randomized trials are needed to define the optimal technique.

Keywords: Breast cancer; Conservative treatment; Boost; Local control; Cosmetic results

\section{Introduction}

The conservative management of the breast cancer knew a development mattering since about twenty years thanks to the screening and to the more and more early diagnosis, to the neoadjuvant treatments, to the improvement of the surgical techniques and especially to the adjuvant radiotherapy [1]. This conservative management has to have 2 purposes, obtain a lowest rate of local recurrence and a satisfactory aesthetic result [2,3]. This challenge justifies the use of oncoplastic surgery techniques that provide both wide excision with clear and sufficient margins and satisfactory aesthetic result and the adjuvant radiotherapy which ensures overall survival, local control and metastasis-free survival similar to those obtained after total mastectomy alone [4,5]. As in the other tumors, it was demonstrated that an increasing dose (boost) in the tumor bed after irradiation of whole breast allows increasing the rate of local control without modifying the free metastasis survival or the overall survival [6]. Various boost techniques are used: the direct beam of electrons, photons by reduced tangential fields or by modulation of intensity, brachytherapy which can be for low, high or pulsed debit rate [7]. 
Citation: Toulba A, Iraqi M, Mouhajir N, Nouh M, Diakité A, et al. (2015) The Additional Irradiation of the Tumor Bed "The Boost" in the Breast Cancer Conservative Treatment: What Techniques?. J Nucl Med Radiat Ther 6: 207. doi:10.4172/2155-9619.1000207

Page 2 of 6

We compare different boost techniques in local control and cosmetic results after breast cancer conservative surgery.

\section{Materials and Methods}

We retrospectively studied all patients at the National Institute of oncology during 2007 treated for a breast cancer and having received a boost on the tumor bed after irradiation of the whole breast.

Eligibility criteria: the histopathologically breast carcinomas classified by the stage I to III according to the TNM classification after conservative surgery.

The criteria of exclusion: the metastatic patients at once, the other histological types than carcinomas, the patients treated previously for a breast cancer and who are admitted for a local recurrence and not exploitable data.

The collected data for each patient were clinical, histological characteristics of tumors and therapeutic.

The data were entered and analyzed by SPSS 13.0. Analytical study was conducted by the exact chi-square test or Fisher test.

\section{Results}

A total of 632 patients were treated for breast cancer. Among these, 74 patients $(65 \%)$ received a complement to irradiation on the tumor bed. The median age was 44.5 years ranging from 25 to 59 years. Hormonal Status: 14 patients (19\%) were postmenopausal (Table 1).

\begin{tabular}{|c|c|}
\hline Characteristics & $N=74$ \\
\hline \multicolumn{2}{|l|}{ Age } \\
\hline$<40$ & $24(32 \%)$ \\
\hline $40-50$ & $40(54 \%)$ \\
\hline$>50$ & $10(14 \%)$ \\
\hline Menopause & $14(19 \%)$ \\
\hline \multicolumn{2}{|l|}{ Location } \\
\hline External quadrant & $50(68 \%)$ \\
\hline Internal quadrant & $14(19 \%)$ \\
\hline \multicolumn{2}{|l|}{ Clinical Tumor stage } \\
\hline $\mathrm{T} 1$ & $28(38 \%)$ \\
\hline $\mathrm{T} 2$ & $44(59 \%)$ \\
\hline \multicolumn{2}{|l|}{ Clinical Nods stage } \\
\hline No & $46(62 \%)$ \\
\hline N1 & $27(37 \%)$ \\
\hline \multicolumn{2}{|l|}{ Histological type } \\
\hline Invasive ductal carcrcinoma & $67(90 \%)$ \\
\hline Medullary carcinoma & $4(5 \%)$ \\
\hline \multicolumn{2}{|l|}{ SBR grade } \\
\hline SBR II & $32(43 \%)$ \\
\hline SBR III & $36(49 \%)$ \\
\hline \multicolumn{2}{|l|}{ Pathological Tumor stage } \\
\hline $\mathrm{T} 1$ & $26(35 \%)$ \\
\hline $\mathrm{T} 2$ & $40(54 \%)$ \\
\hline \multicolumn{2}{|l|}{ Pathological Nods stage } \\
\hline \multirow[t]{2}{*}{ No } & $35(47 \%)$ \\
\hline & $20(27 \%)$ \\
\hline Intraductal component & $28(39 \%)$ \\
\hline
\end{tabular}


Citation: Toulba A, Iraqi M, Mouhajir N, Nouh M, Diakité A, et al. (2015) The Additional Irradiation of the Tumor Bed "The Boost" in the Breast Cancer Conservative Treatment: What Techniques?. J Nucl Med Radiat Ther 6: 207. doi:10.4172/2155-9619.1000207

Page 3 of 6

\begin{tabular}{|l|l|}
\hline LVI + & $21(28 \%)$ \\
\hline ER/PR+ & $60(81 \%)$ \\
\hline Chemotherapy & \\
\hline Neoadjuvant & $2(3 \%)$ \\
\hline Adjuvant & $65(88 \%)$ \\
\hline Boost technics & \\
\hline Electrons & $28(38 \%)$ \\
\hline Brachytherapy & $28(38 \%)$ \\
\hline Phontons & $18(24 \%)$ \\
\hline
\end{tabular}

Table 1: Patient/tumor characteristics.

\section{The Characteristics of Tumors (Table 1)}

\section{Clinical characteristics}

Location of the tumor: The tumor was at the external quadrants in 50 cases (68\%), at the internal quadrants in 14 cases (19\%) and at the central quadrant in 1 case. The location was not specified in 3 cases (4\%).

- Clinical T Stage: 72 patients (97\%) were classified as T1 and T2 with 28 cases of $\mathrm{T} 1(38 \%)$ and 44 cases of $\mathrm{T} 2(59 \%)$, one case of $\mathrm{T} 3$ and one of T4.

- Clinical N stage: 46 patients (62\%) were classified clinically N0. 27 patients (37\%) were classified clinical N1.

\section{Histological characteristics of tumors}

Histological Types: the most common histological type was the invasive ductal carcinoma, found to 67 cases (90\%). Other histological types were rare: 4 cases of medullary carcinoma (5\%), 1 case of papillary carcinoma, 1 case of mucinous carcinoma and one case of carcinosarcoma.

The histological grade of SBR: SBR grade III was predominant in 36 cases (49\%) followed by grade II in 32 cases (43\%) while the SBR grade I was found in only 6 cases (8\%).

The histological tumor size: 49 tumors $(66 \%)$ were less than $30 \mathrm{~mm}$ with $5(7 \%)$ were less than $10 \mathrm{~mm}$.

Pathological N Stage: in axillary lymph nodes dissection, 35 patients (47\%) were pN0 and 20 cases (27\%) were classified as pN1.

Intraductal component: was found in 28 cases (39\%)

Lymphovascular invasion (LVI): was present in 21 cases (28\%)

Hormone receptors (estrogen, progesterone): were positive in 60 cases $(81 \%)$.

Human Epidermal Growth Factor Receptor-2 (HER2) status: was positive in 5 cases $(7 \%)$, negative in 44 patients $(60 \%)$ and unknown in 25 cases (33\%).

\section{Treatment}

Neoadjuvant chemotherapy was performed in 2 cases (3\%) and the number of cures ranged from 3 to 4 cycles.

Type of the conservative surgery: lumpectomy in 54 cases (72\%), quadrantectomy in 19 cases (23\%) and zonectomy in a single case. The oncoplastic or the remodeling was performed in 24 patients $(32 \%)$. Repeat excision was performed in 19 patients (25\%) for positive margins in 12 cases (63\%) and close margins in 7 cases (37\%).

Adjuvant chemotherapy: 65 patients (88\%) received adjuvant chemotherapy. It was made in a pattern with anthracyclines in 46 patients (71\%) while 11 patients (17\%) received a sequential Anthracyclines / Taxane. The chemotherapy regimen was not specified in 8 patients $(12 \%)$.

\section{Radiotherapy}

The target volume: The breast and the chest wall were irradiated in all patients (100\% of cases), the supraclavicular fossa in 41 cases (55\%), the internal mammary chain in 29 cases (39\%), the axilla in 6 cases (8\%) for an insufficient lymph node dissection $(<10$ lymph node removed).

\section{Dose / fractionation}

- Standard fractionation, $50 \mathrm{~Gy}$ in 25 fractions of $2 \mathrm{~Gy}$ in 53 patients (72\%).

- Hypofractionation, 42 Gy in 15 fractions of 2.8 Gy in 21 patients (28\%).

- The median of overall treatment time: 37 days.

\section{The additional irradiation of the tumor bed or "Boost"}

Techniques: Electrons and brachytherapy each performed in 28 patients $(38 \%)$ and the additional photons in 18 patients $(24 \%$ of the cases). For the brachytherapy, it was HDR in 20 cases (71\%) and LDR in only 8 cases $(29 \%)$.

Delay: the delay between the end of the irradiation of the breast and the boost was 11 days. This period varies depending on the boost technique: it is of 6 days for electrons, of 5 days for photons while for the brachytherapy, this period was much longer than 22 days. 


\section{Dose}

- Electrons: 15 Gy in 5 fractions of $3 \mathrm{~Gy}$.

- Photons: 15 Gy in 5 fractions of 3 Gy for half of the cases or in 6 fractions of $2.5 \mathrm{~Gy}$ in the other half.

\section{Brachytherapy}

LDR: $15 \mathrm{~Gy}$

HDR: 2 fractions of 5 Gy separated by 10 to 12 hours.

\section{The follow-up}

The median follow-up was 52 months with a range of 3 to 47 months: 61 patients $(82.5 \%)$ were in free-locale recurrence, three patients $(4 \%)$ had a local recurrence. In two patients, this recurrence was isolated while in the third, it was associated with metastatic relapse. Distant metastases were found in 10 patients (13.5\%). Among these, four are always followed, two died and four were lost-of-view. In one patient, a contralateral breast cancer was diagnosed. 9 patients (12\%) were lost to follow-up.

\section{The local recurrences}

During the follow-up, three local recurrences were diagnosed among. The time of the occurrence of the local recurrence was short for two patients (6 months). All the local relapses were located in the tumor bed. Recurrences were associated with permeation nodules and lung metastases in a patient. Among these patients with a local recurrence, one has benefited once again conservative treatment while in the second, a radical surgery was necessary (Table 2).

\begin{tabular}{|l|l|l|l|l|}
\hline & Brachytherapy & Electrons & Photons & P-value \\
\cline { 2 - 4 } & $\mathrm{n}(\%)$ & $\mathrm{n}(\%)$ & $\mathrm{n}(\%)$ & \\
\hline $\begin{array}{l}\text { Locale recurrence } \\
(\mathbf{N}=\mathbf{3})\end{array}$ & $1(33.3 \%)$ & $1(33.3 \%)$ & $1(33.3 \%)$ & $>0.05$ \\
\hline Fibrosis (N=28) & $14(50 \%)$ & $8(29 \%)$ & $6(21 \%)$ & $>0.05$ \\
\hline $\begin{array}{l}\text { Pigmentation } \\
\text { disorders (N=1)1) }\end{array}$ & $6(55 \%)$ & $3(18 \%)$ & $2(18 \%)$ & $>0.05$ \\
\hline Telangiectasis (N=5) & $4(80 \%)$ & $1(20 \%)$ & & $>0.05$ \\
\hline
\end{tabular}

Table 2: Occurrence of significant events (local recurrence and side effects).

\section{The side effects}

1. Fibrosis: fibrosis was noted in 28 patients (38\%). The site was the tumor bed in 22 cases (78\%), the entire breast in 4 cases $(14 \%)$. The degree of fibrosis was mild in 11 cases $(78 \%)$, moderate in 11 cases

2. Telangiectasis: observed in 5 cases (7\%).

3. Disorder of pigmentation: found in 11 cases (15\%).

\section{The side effects depending on the boost technique:}

There were more side effects in patients who received a brachytherapy boost with a fibrosis rate of 50\% (14 cases) compared with photons $21 \%$ (6 cases) and $29 \%$ with electrons (8 cases). Pigmentation disorders were observed in $55 \%$ of cases (6 patients) treated by brachytherapy, in 18\% ( 2 cases) and 27\% (3 cases) of those treated by photons and electrons respectively. As for telangiectasis, they were found only in patients treated by brachytherapy $80 \%$ ( 4 cases), and by electrons $3 \%$ (1 patient) Table 2 .

\section{Discussion}

The conservative treatment has gradually become the standard treatment for the breast cancer; it is proposed in the great majority of the patients, either immediately or after a neoadjuvant treatment when the volume of the tumor and/or the breast does not allow initially a conservative surgery. This treatment is breast conservative surgery, axillary nodes dissection and irradiation of the preserved breast with boost. The overall survival is similar to that obtained by the mastectomy, several randomized trials was demonstrated in, a low risk of local recurrence with adjuvant radiotherapy which reduces the risk of local recurrence to less than $66 \%$ [1] and a satisfactory aesthetic result, made possible by the progress of the oncoplastic surgery [8].

Interest of the boost: the rational of the increase of dose in the tumor bed is based on several observations: most of the ipsilateral breast recurrences sit in or very close to the primitive site (in $67-100 \%$ of the cases) and the residual microscopic foci after lumpectomy are found around this tumor bed [9]. Therefore, it was shown in several trials, that an increase of dose in the tumor bed after irradiation of the totality of the breast, allows to increase the rate of local control ( $41 \%$ in 5 years) without modifying the metastasis-free survival or overall survival $[5,10]$. This benefit of the boost in local control decreases with age [11].

\section{In our study all local relapses were located in the tumor bed:}

Location of the tumor bed: the delineation of the tumor bed is a necessary step in the optimization of the conservative treatment of the breast tumors. It requires a multidisciplinary approach. Until these last years, radiotherapists were confronted with important difficulties in the location of the tumor bed. The introduction of surgical clips coupled with the realization of a CT scan in position of treatment allowed an improvement of the volume's tracking overlay. However, most of the authors agree to recognize the limits of this conventional method in identifying the tumor bed. Among the problems encountered in the location of the tumor bed are gaps between the pectoral clip and the zone of high density associated with the clips. These gaps can be explained by the position of treatment which differs from the operating position [11]. Moreover, the oncoplastic techniques engendered new challenges. The Anglo-Saxon approach in the definition of the tumor bed is less difficult in this context because most of the other authors use the postoperative cavity [12]. The location of this surgical cavity is made by clips [13] or from the data of the preoperative MRI [14] or ultrasound [15]. Some authors proposed a standardized method for the location of the tumor bed [11].

\section{The techniques of the boost}

Various techniques are used to deliver the boost to the tumor bed: the direct beam of electrons, photons with reduced tangential fields, photons with conformal 3D-radiotherapy and brachytherapy. The choice of the technique depends on the experience of the center, on the preference of the radiotherapist and also the specific parameters of tumors and on patients. The relative use of the different techniques varies considerably between centers. In the trial EORTC 22881/10882 ' boost versus no boost ', the boost by electrons was used at $59 \%$ of the patients in the Netherlands and brachytherapy in $8 \%$. In France, the 
Page 5 of 6

brachytherapy was applied in $11 \%$ of the cases. In Belgium, the boost by photons was rarely practiced [5]. During the period of this study, the relative use of photons and particularly that of the interstitial brachytherapy gradually decreased from 30 to $25 \%$ and from 14 to $4 \%$ respectively, along with an increase of the use of electrons from 54 to $69 \%$. It can be explained by the modernization of the radiotherapy equipments and the acquisition of linear accelerators which offer a wide range of electron energy and also by the heaviness of the infrastructure of the brachytherapy, its measures of radioprotection, the training of the practitioners whom it requires, the time consumed by the radiotherapists and the medical physicists, what limits the practice of this technique.

\section{Comparison of the Techniques of the Boost}

\section{The volume}

The boosted median volume varies considerably with the used technique, so, this volume is the most reduced in the interstitial brachytherapy $\left(60 \mathrm{~cm}^{3}\right)$, it is more than twice upper in the boost by electrons $\left(144 \mathrm{~cm}^{3}\right)$ and it is five times for photons $\left(288 \mathrm{~cm}^{3}\right)$ [7].

\section{The overall duration of the irradiation}

The additional external radiotherapy (photons or electrons) is usually started immediately without any delay after breast irradiation for the majority of the patients, while a median time of 18 days was noted between the end of the irradiation and start of the boost by brachytherapy in the EORTC trial which is responsible for a considerable increase in the overall duration of treatment for this group of patients [16]. In our study, this period is 22 days.

\section{Local Control}

A number of studies comparing different techniques of boost of the tumor bed were published. In terms of comparative efficiency between the techniques of the boost, the analysis of the literature found only retrospective studies with the exception of the randomized trial of the Curie's Institute which compared, in patients not operated, a boost by telecobaltherapy with a boost by brachytherapy for low dose and found a local recurrence rate at five years significantly lower in patients treated by brachytherapy (30 against 16\%, p<0.03) [12]. However, the vast majority of published studies comparing electron boost and boost LDR brachytherapy showed no significant difference in the rate of local control. The study of literature concerning the comparative efficiency between boost by brachytherapy (HDR) and by electrons found two studies, of which one randomized [17]. This study is a trial with double randomization, the first being made on the realization or not of the boost; the following is based on the technique of the boost (electrontherapy against brachytherapy HDR). Only the analysis of the series of Hammer and coll. found efficiency significantly better in patients who received brachytherapy HDR delivered a fraction of 10 Gy [18]. Indeed, besides the retrospective nature of these series, some of these studies show a significant bias mainly due to the age of the patients between the two groups. Indeed, the patients taken care by brachytherapy are significantly younger than those treated by electrons $[18,19]$. In our study, there is no difference in local recurrence for the different techniques.

\section{Aesthetic results}

The comparison of the rates of aesthetic results between electron boost or brachytherapy LDR is difficult to interpret. Indeed, six published retrospective studies was not found in four of them, a significant difference between the two techniques of radiation boost to the tumor bed [20-22]. It was found in the study of Touboul and coll. best results with the boost by electrons ( 83 against $62 \%, \mathrm{p}<0.001$ ) [21] and in that of Wazer and coll. with the brachytherapy (68 against $90 \%$, $\mathrm{p}<0.001)$ [20].

For the comparison of the rates of aesthetic results between the electron boost or brachytherapy HDR, only Hammer and coll. found an aesthetic result significantly better with the brachytherapy (HDR) (70 against $88 \%, \mathrm{p}<0.0001$ ) [18], while the team of National Institute of Oncology of Budapest found same results [17]. Whatever is the considered dose rate, the best aesthetic results found in case of boost by brachytherapy can be explained by the "sub-dosage" of the skin surface (safety margin of $5 \mathrm{~mm}$ between the most superficial vectors and the skin) [23].

The fibrosis is the most common side effect and it is compromises the aesthetic results. Its occurrence, as well in the tumor bed as in the whole of the breast, was similar for the three techniques in the EORTC trial [11]; $12 \%$ of the patients developed moderate to severe fibrosis in whole breast and $25 \%$ at the primary site of the tumor. The dose and the volume are not the only factors that may affect the cosmetic results, so, the technique of initial irradiation of the breast should not be neglected. A randomized trial showed in 2 years a change of appearance of the breast in 52\% of women after two-dimensional conventional irradiation compared with $36 \%$ when the radiotherapy with modulation of intensity is used to maximize the homogeneity of the breast irradiation [24]. In our study, there is no difference for the different techniques.

\section{What techniques?}

The analysis of the results of phase II trials and some phase III trials have not shown significant superiority of the one or the other technique both in terms of local control and side effects, the choice of the technique of boost depends on the experience of the center and technical equipment. The brachytherapy, allowing delivering a high dose, in a short time and in a reduced volume, seems to be adapted to the treatment of the target volumes profoundly placed, in voluminous breasts thanks to a better distribution of dose. Compared to high energy electrons or photons by reduced tangential fields, this technique allows to reduce both the skin dose and the volume receiving a high dose and this thanks to a better conformation and underdosing of the superficial tissues. Once the necessary, energy to cover a deep target volume exceeds $9 \mathrm{MeV}$, the skin dose increases and the risk of telangiectasia exceeds $10 \%$ [25]. On the other hand, if the target volume is superficial or if the volume of boost for the brachytherapy is less than $5 \mathrm{~mm}$ of the surface, the rate of telangiectasia rise [26]. Intensity-modulated radiation therapy (IMRT) allows in these cases to save the skin tissue by providing a focal and deep irradiation [27], but no comparison was published in this context. The choice of the technique depends on the experience of the center, on the preference of the radiotherapist and also on the patient and the specific parameters of the tumor.

With an equal efficacy and toxicity, the choice of a treatment should also consider the cost and the quality of life of patients. These two parameters did not make, in our study. 


\section{Conclusion}

The increase of dose in the tumor bed after conservative treatment of the breast cancer allows increasing the rate of local control without compromising too much the aesthetic results. Different techniques can be used to deliver this boost. The comparison between these different techniques showed no significant differences both for the local control and for the aesthetic results. There is a necessity of randomized trials comparing these techniques to be able to draw any definitive conclusions for the choice of the optimal technique.

\section{Conflicts of Interests}

The authors declare to have no conflicts of interests.

\section{References}

1. Clarke M, Collins R, Darby S, Davies C, Elphinstone P, et al. (2005) Effects of radiotherapy and of differences in the extent of surgery for early breast cancer on local recurrence and 15-year survival: an overview of the randomised trials. Lancet 366: 2087-2106.

2. Hau E, Browne L, Capp A, Delaney GP, Fox C, et al. (2013) The impact of breast cosmetic and functional outcomes on quality of life: long-term results from the St. George and Wollongong randomized breast boost trial. Breast Cancer Res Treat 139: 115-123.

3. Hau E, Browne LH, Khanna S, Cail S, Cert G, et al. (2012) Radiotherapy breast boost with reduced whole-breast dose is associated with improved cosmesis: the results of a comprehensive assessment from the St. George and Wollongong randomized breast boost trial. Int J Radiat Oncol Biol Phys 82: 682-689.

4. Dobke M (2012) Impact of Advances in Breast Cancer Management on Reconstructive and Aesthetic Breast Surgery. Clin Plast Surg 39: 465-475.

5. Bartelink H, Horiot JC, Poortmans P, Struikmans H, Van den BogaertW, et al. (2001) European Organization for Research and of Cancer Radiotherapy and Breast Cancer Groups. Recurrence Rates after Treatment of Breast Cancer with Standard Radiotherapy with or without Additional Radiation. N Engl J Med 345: 1378-1387.

6. Bartelink H, Horiot JC, Poortmans P, Struikmans H, Van den Bogaert W, et al. (2005) Impact of a higher radiation dose on local control and survival in breast-conserving therapy of early breast cancer: 10-year results of the randomized boost versus no boost EORTC 22881-10882 trial. J Clin Oncol 25: 3259-3265.

7. Poortmans P, Bartelink H, Horiot JC, Struikmans H, Van den Bogaert W, et al. (2004) The influence of the boost technique on local control in breast conserving treatment in the EORTC 'boost versus no boost' randomised trial. Radiotherapy and Oncology 72: 25-33.

8. Doré M, Hennequin C (2012) Late sequelae and cosmetic outcome after radiotherapy in breast conserving therapy. Cancer Radiother 16: 462-469.

9. Holland R, Connolly JL, Gelman R (1990) The presence of an extensive intraductal component (EIC) following a limited excision predicts for prominent residual disease in the remainder of the breast. J Clin Oncol 8:113-118.

10. Romestaing P, Lehingue Y, Carrie C, Coquard R, Montbarbon X, et al. (1997) Role of a 10 Gy boost in the conservative treatment of early breast cancer: results of a randomized clinical trial in Lyon. France. J Clin Oncol 15: 963-968.

11. Oden S, Thureau S, Baron M, Hanzen C (2010) Traitement conservateur du cancer du sein : optimisation du repérage du lit tumoral. Cancer/ Radiothérapie 14: 96-102.
12. Van der Laan HP, Dolsma WV, Maduro JH, Korevaar EW, Hollander M, et al. (2007) Three-dimensional conformal simultaneously integrated boost technique for breast-conserving radiotherapy. Int J Radiat Oncol Biol Phys 68: 1018-1023.

13. Weed DW, Yan D, Martinez AA, Vicini FA, Wilkinson TJ, et al. (2004) The validity of surgical clips as a radiographic surrogate for the lumpectomy cavity in image-guided accelerated partial breast irradiation. Int J Radiat Oncol Biol Phys 60: 484-492.

14. Whipp EC, Halliwell M (2008) Magnetic resonance imaging appearances in the postoperative breast: the clinical target volume-tumor and its relationship to the chest wall. Int J Radiat Oncol Biol Phys 72: 49-57.

15. Coles CE, Cash CJ, Treece GM, Miller FN, Hoole AC, et al. (2007) High definition three-dimensional ultrasound to localise the tumour bed: a breast radiotherapy planning study. Radiother Oncol 84: 233-241.

16. Fourquet A, Campana F, Mosseri V, Cetingoz R, Luciani S, et al. (1995) Iridium-192 versus cobalt- 60 boost in $3-7 \mathrm{~cm}$ breast cancer treated by irradiation alone: final results of a randomized trial. Radiother Oncol 34: 114-120.

17. Polgar C, Fodor J, Orosz Z, Major T, Takacsi-Nagy Z, et al. (2002) Electron and high-dose-rate brachytherapy boost in the conservative treatment of stage I-II breast cancer first results of the randomized Budapest boost trial. Strahlenther Onkol 178: 615-623.

18. Hammer J, Seewald DH, Track C, Zoidl JP, LabeckW (1994) Breast cancer: primary treatment with external-beam radiation therapy and highdose- rate iridium implantation. Radiology 193: 573-577.

19. Touboul E, Belkacemi Y, Lefranc JP, Uzan S, Ozsahin M, et al. (1995) Early breast cancer: influence of type of boost (electrons vs iridium-192 implant) on local control and cosmesis after conservative surgery and radiation therapy. Radiother Oncol 34: 105-113.

20. Wazer DE, Kramer B, Schmid C, Ruthazer R, Ulin K, et al. (1997) Factors determining outcome in patients treated with interstitial implantation as a radiation boost for breast conservation therapy. Int J Radiat Oncol Biol Phys 39: 381-393.

21. Mansfield CM, Komarnicky LT, Schwartz GF, Rosenberg AL, Krishnan L, et al. (1995) Ten-year results in 1070 patients with stages I and II breast cancer treated by conservative surgery and radiation therapy. Cancer 75 : 2328-2336.

22. Perez CA, Taylor ME, Halverson K, Garcia D, Kuske RR, et al. (1996) Brachytherapy or electron beam boost in conservation therapy of carcinoma of the breast: a nonrandomized comparison. Int J Radiat Oncol Biol Phys 34: 995-1007.

23. Hannoun-Lévi JM, Marsiglia H (2004) Complément d'irradiation par curiethérapie dans le cancer du sein : que savons-nous ? Où allons-nous? Cancer/Radiothérapie $8: 248-254$.

24. Yarnold JR, Donovan E, Bleakley N (2002) Randomised trial of standard $2 \mathrm{D}$ radiotherapy (RT) versus $3 \mathrm{D}$ intensity modulated radiotherapy (IMRT) in patients prescribed breast radiotherapy. Radiother Oncol 64: 15.

25. Turesson I, Notter G (1984) The influence of fraction size in radiotherapy on the late normal tissue reaction (I C II). Int J Radiat Oncol Biol Phys 10: 593-606.

26. Van Limbergen, Pescova-Georg P (2000) The source-skin distance measuring bridge (SSMB) reduces indeed skin telangiectasia after interstitial boost in breast conserving therapy (BCT) for breast cancer: 15 years of clinical experience. Radiother Oncol 55: 32.

27. Graham P, Fourquet A (2006) Placing the Boost in Breast-conservation Radiotherapy: A Review of the Role, Indications and Techniques for Breast-boost Radiotherapy. Clinical Oncology 18: 210-219. 